\title{
RELATIONSHIPS OF MEAN CORPUSCULAR VOLUME WITH DIAMETER AND SURFACE AREA OF CANINE ERYTHROCYTES
}

\author{
Indira Silva ${ }^{1}$, K. Thananjayan ${ }^{1}$, DRA Dissanayake ${ }^{1}$, WCR Fernando ${ }^{2}$ and \\ M. Murugananthan ${ }^{3}$ \\ ${ }^{1}$ Veterinary Teaching Hospital, University of Peradeniya, Sri Lanka \\ ${ }^{2}$ Department of Animal Production and Health, Sri Lanka \\ ${ }^{3}$ Department of Parasitology, Faculty of Medicine, University of Jaffna, Sri Lanka
}

\begin{abstract}
SUMMARY: Assessment of erythrocyte morphology is an important aid in diagnostic haematology. Anisocytosis which is changes in RBC size, correspond to changes in diameter and surface area (SA) of the cell, and are not always reflected in the Mean Corpuscular volume (MCV). This paper discusses the relationship of MCV with surface area and diameters (vertical and horizontal) in $2 D$ views of erythrocytes of clinically healthy dogs using confocal microscopy. This information would be valuable for early detection of cellular changes in dogs in subclinical or clinical diseases.

The average diameters, average $S A$, and MCV values of RBCs studied were in normal distribution and the mean values of $7.169 \pm 0.648 \mu \mathrm{m}$ for horizontal diameter (D1), $7.1245 \pm 0.6646 \mu \mathrm{m}$ for vertical diameter (D2), and $41.061 \mathrm{~m}^{2} \pm 6.866$ for SA did not reveal a significant correlation or a strength of association with the MCV values, indicating that the MCV value has limitations as an objective measurement of detecting anisocytosis.
\end{abstract}

\section{INTRODUCTION}

Examination of blood is virtually the universal first step in the evaluation of health and disease in human and animal patients as physiological and pathological changes in most tissues are reflected directly or indirectly in blood. Erythrocyte morphology is routinely assessed microscopically and is an important aid in diagnostic haematology (Thrall, 2006; Jain, 1993; Proctor et al, 1976). The morphology of red cells is categorized according to colour, size, shape, intra or epicellular structures, and their arrangement on blood films, which are unique for each animal species (Ford, 2013; Thrall, 2006).

The typical 3D biconcave disk shape of RBCs in animal species show very little variability in size and shape between cells (Adili et al., 2017). Anisocytosisis defined as size variations resulting from microcytes, macrocytes or both (Proctor et al, 1976). Parameters of red cell volumes, such as, Mean Corpuscular Volume (MCV), Red Cell Distribution Width (RDW) and/or RDW-SD are tools generally used to quantify anisocytosis. Changes in RBC size correspond to changes in cell diameter and not necessarily to changes in RBC volume, and changes in cell volumes are not always reflected in the MCV. Furthermore, the surface area and volume changes in disease do not occur equally in all RBCs in a sample (Jay and Rowlands, 1975). Hypochromic microcytic erythrocytes in irondeficiency show decreased MCV when determined electronically, but show normal diameter (Thrall, 2006). The small diameter of spherocytesis not reflected in the MCV value as the volume of spherocytes is always within the reference range (Thrall, 2006). Presence of schistocytes in blood film is not reflected in the MCV value. The RDW is more sensitive than MCV to detect erythrocyte sub populations during response to irondeficiency anemia (Jain, 1993). However, a significant proportion of human patients with RBC macrocytosis due to pernicious anemia had normal RDW rather than an elevated RDW (Perry et al, 1985). Furthermore, red cell agglutination can falsely elevate MCV values, and even falsely lower HCT measurement as the upper limit in automated analyzers is usually 360fl, thereby excluding counting large RBC clumps (Greer, 2009). Immature RBCs and oxidative damage due to metabolic diseases and conditions can be detected only by examining erythrocyte morphology (Udroiu, 2014). Erythrocyte morphometry provide valuable data on changes in erythrocyte morphology and could complement the results obtained by standard hematology methods. For example, morphometric studies have demonstrated that the erythrocytes in male rabbits are smaller than in females, though the average cell volume was not significantly different (PoljičakMilasetal, 2009).

Changes in diameter may not be reflected in the MCV obtained as a direct measurement from automated hematology analyzers, which is the average volume of RBCs in a mixture of cells (Weiss and Wardrop, 2010), and does not indicate individual changes in RBC sizes. Therefore, it is important and timely to study morphometry of RBCs, such as, the relationship between the diameter, the surface area (SA) and the MCV of individual RBCs. Several techniques have been proposed for morphological studies of biological specimens, such as optical coherent tomography and confocal microscopy. The objective of this study was to analyze the relationship of MCV with SA and diameters in $2 \mathrm{D}$ views of RBCs of clinically healthy dogs using

Corresponded auther: Indira Silva, indiradush@gmail.com

https://orcid.org/0000-0001-7210-8457 
confocal microscopy. The information presented would be valuable for early detection of cellular changes in dogs with subclinicalor clinicaldiseases.

\section{MATERIALS AND METHODS}

Dogs presented to Veterinary Teaching Hospital (VTH), Peradeniya with normal Full Blood Counts (FBC) analyzed using a fully automated veterinary hematology analyzer (MS9-5V, MELET Schloesing laboratories, France) were selected for this study. A volume of $1 \mathrm{ml}$ blood was collected to $\mathrm{K}_{2}$ EDTA coated tubes with the consent of the owners. The anticoagulant $\mathrm{K}_{2}$ EDTA is recommended by ICSH (International Council for Standardization in Hematology) and CLSI (Clinical and Laboratory Standards Institute) as the choice of anticoagulant for blood cell counting.

Blood smears were prepared from each sample, stained with Leishman stain and transported to the Parasitology laboratory, Faculty of Medicine, University of Jaffna to study morphometry of RBC in $2 \mathrm{D}$ view using a Laser scanning confocal microscope (Carl Zeiss LSM 700, Germany).Up to 500 RBCs in the middle one third of the monolayers of each slide were randomly examined by cross sectional method under oil immersion $(\times 100)$ using the above microscope. A laser beam (405 nm -639 nm wavelength) was passed through the confocal optical path, and images were obtained with Zeiss Axio Imager Z2 (high resolving and high sensitive cameras) on the monitor at $2560 \times 1920$ resolution to generate the 2dimensional object information of the RBCs (Cortada, 2013; Bernhard, 2012 in LSM 700 Operating Manual Black edition 2012). The digital images were acquired at $2560 \times 1920$ resolution using a Laser scanning confocal microscope (Carl Zeiss LSM 700, Germany) to measure perpendicular diameters (horizontal, D1 and vertical, D2) and surface area (SA) of each RBC in micrometer using the ZEN lite (blue edition) software from Carl Zeiss. The entire system was controlled through a standard high-end Personnel Computer (PC) with Windows operating system. The approximate perpendicular diameters (horizontal, D1 and vertical, D2) and surface area (SA) of each $\mathrm{RBC}$ was measured in micrometers up to 3 decimals using the ZEN lite (blue edition) software from Carl Zeiss. These values were analyzed with the MCV values in the FBC report.

\section{Data analysis}

Anderson Darling normality test (ADNT) was performed using 'MINITAB' software to analyze whether the data obtained for MCV, diameters and SA of RBCs follows a normal distribution (Ghasemi and Zahediasl, 2012). Mean diameter, standard deviation and variance, minimum, maximum data for D1, D2 and SA of $500 \mathrm{RBC}$ per sample were calculated using the 'MINITAB' software. The Pearson Correlation Coefficient test was performed using the same software to assess the strength and direction of association of MCV values with diameters (D1, D2, average diameters) and SA individually. The Linear regression test was performed to predict the value of the dependent variable (MCV) based on the value of the independent variable (SA, D1 and D2). Box plots were created separately to visualize the pattern of association between the MCV, D1, D2 and SA using the Box plot R software.

\section{RESULTS AND DISCUSSION}

Morphometry provides higher reliability and reproducibility of cytological and histopathological diagnoses in human medicine (Albertini et al., 2003). This paper compares the relationship of MCV with surface area (SA) of erythrocytes and diameters in $2 \mathrm{D}$ views of RBCs. The Full Blood Count (FBC) on blood collected from 14 apparently healthy dogs ( 9 females, 5 males) of different breeds (German shepherd, Lion shepherd, and Rottweiler, Doberman and Cross breds), with varying ages (4 months to 13 years) were measured within 4 hours of collection.

The perpendicular diameters (horizontal, D1 and vertical, D2) and surface area (SA) of each RBC in micrometer up to 3 decimals using the ZEN lite (blue edition) software from Carl Zeiss are shown in Figure 1.The above software allowed accurate morphometric measurements, similar to other software, such as OPTIKATM Vision Pro software (Adili et al, 2017), than the conventional method of measuring the diameters of erythrocytes in a blood film using a scale on an oil immersion lens in a light microscope. The use of software reduces errors due to the human factor that is involved in studies with ocular micrometer.

The reference values of MCV for dogs in Sri Lanka range between 52-76 fl and 60-77 fl (Silva and Mallawa, 2010). The Anderson Darling normality test revealed that the average diameter, average $\mathrm{SA}$, and $\mathrm{MCV}$ values of RBCs were in normal distribution $(\mathrm{P}=0.075,0.198$, and 0.135 , respectively). The mean diameters of 7.169 $( \pm 0.648 \mu \mathrm{m})$ for $\mathrm{D} 1,7.1245( \pm 0.6646 \mu \mathrm{m})$ for $\mathrm{D} 2$, and $41.061 \mu \mathrm{m}^{2}( \pm 6.866)$ for SA calculated were similar to values obtained by Adili et al, (2017) for diameter (6.29$7.77 \mu \mathrm{m})$ and surface area $\left(25.55-47.05 \mu \mathrm{m}^{2}\right)$ of RBCs of Sloughi and German Shepherd dogs.

The Pearson Correlation coefficient did not reveal significant relationships between $\mathrm{MCV}$ and $\mathrm{SA}(\mathrm{r}=$ 0.023, $\mathrm{p}=0.140), \mathrm{MCV}$ and D1 $(\mathrm{r}=0.011, \mathrm{p}=0.140)$, or between $\mathrm{MCV}$ and $\mathrm{D} 2(\mathrm{r}=-0.05, \mathrm{p}=0.001)$. Linear regression analysis between $\mathrm{MCV}$ (dependent variable) and SA did not reveal a significant correlation ( $\mathrm{r}-0.011$, $\mathrm{p}=0.140$ ), or a strength of association (P-0.474, R-sq. $0.1 \%$ ). A significant linear relationship or significant strength of association was not evident between MCV and D1(p-0.474, R-sq. $-0.0 \%$ ) or between MCV and D2 ( $p=0.001$, R-sq. $0.1 \%$ ). The box plots also did not reveal a pattern of association between the MCV and SA (Figure 2) or between MCV and D1 (Figure 3) or D2 (Figure 4).Adili et al, 2017 also have not seen significant differences between the diameter and the SA of RBCs of dogs.

Anisocytosis in a blood sample, depicted by parameters of red cell volumes, such as, MCV and RDW do not complement the changes in RBC size in relation to diameter or surface area (Proctor et al, 1976). Our results 


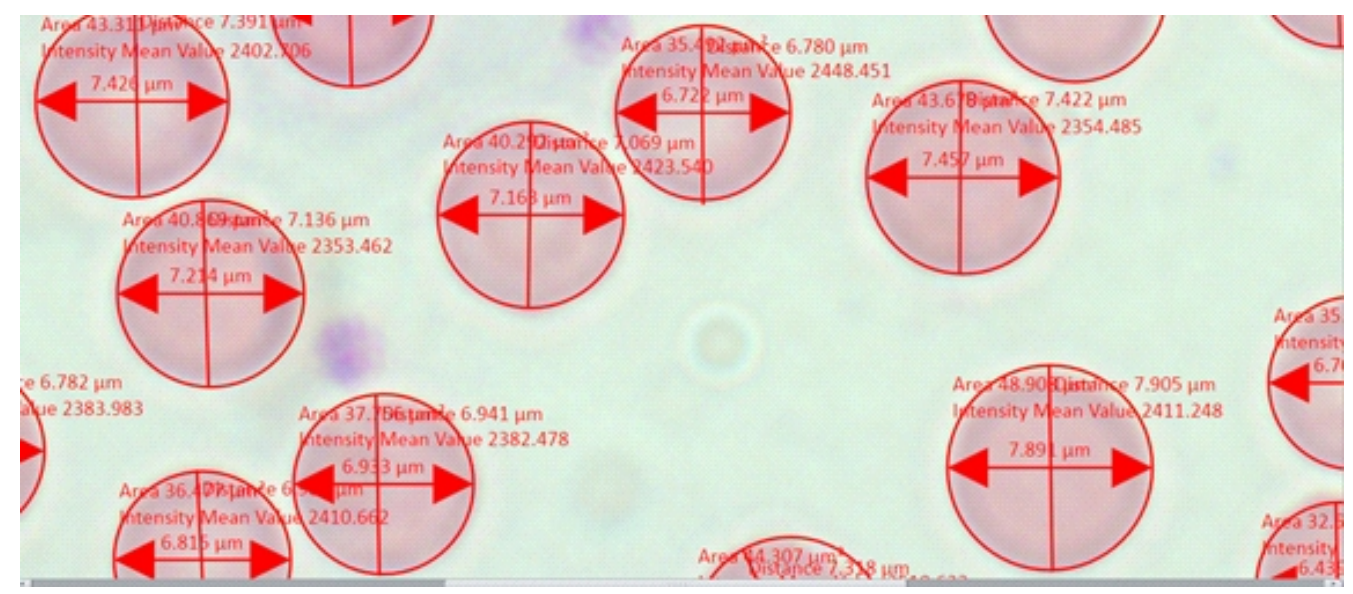

Figure 1. Perpendicular diameters (horizontal, D1 and vertical, D2) and surface area of RBC

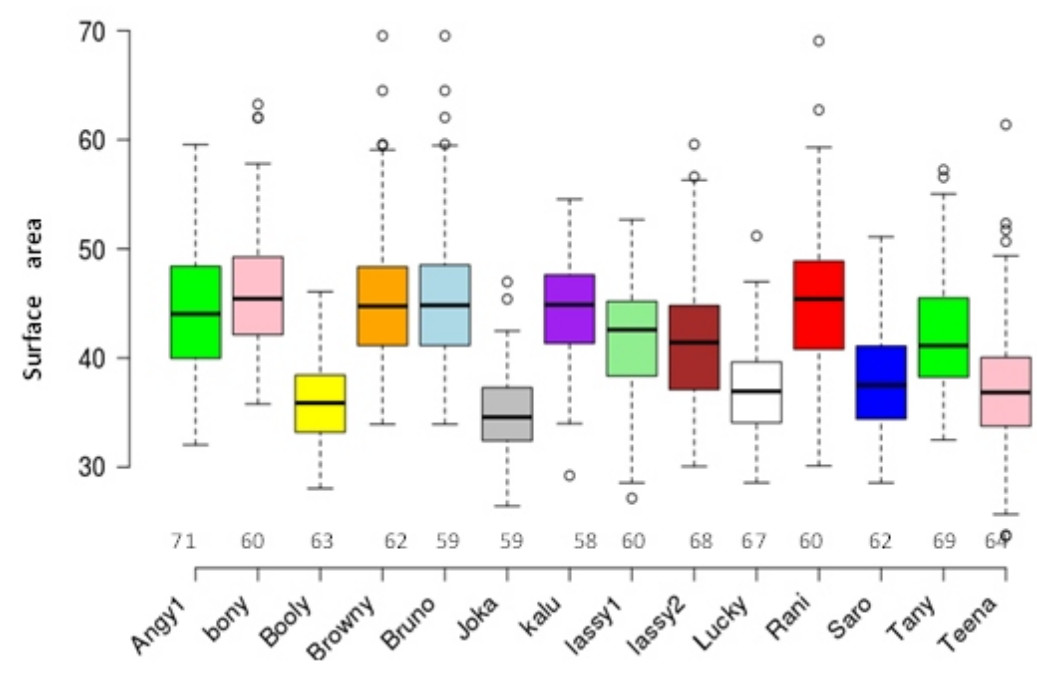

Figure 2. Variation of surface area of red blood cells in dogs

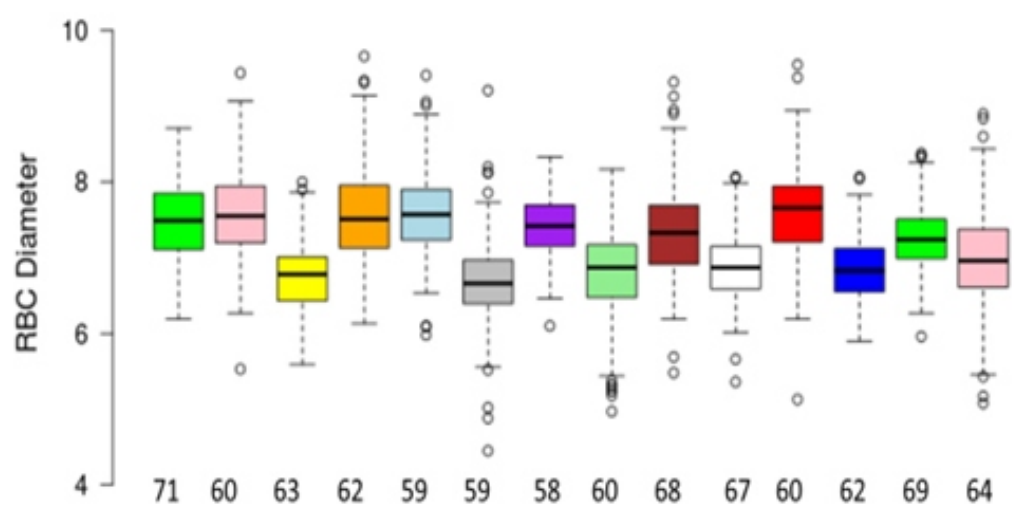

Figure 3. Variation of horizontal diameter of red blood

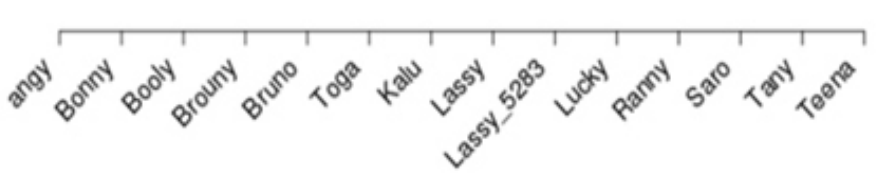

show that diameters and surface areas of RBCs do not correspond with the MCV values calculated by the automated analyzer, indicating that the MCV value has limitations as an objective measurement of detecting anisocytosis. Practitioners may interpret changes in test results as significant, when in reality, they are close to reference range, and they also should be able to recognize changes in a patient's test results that are due to random error before the test results are reported (Houwen, 1989). Electronic hematology analyzers are 


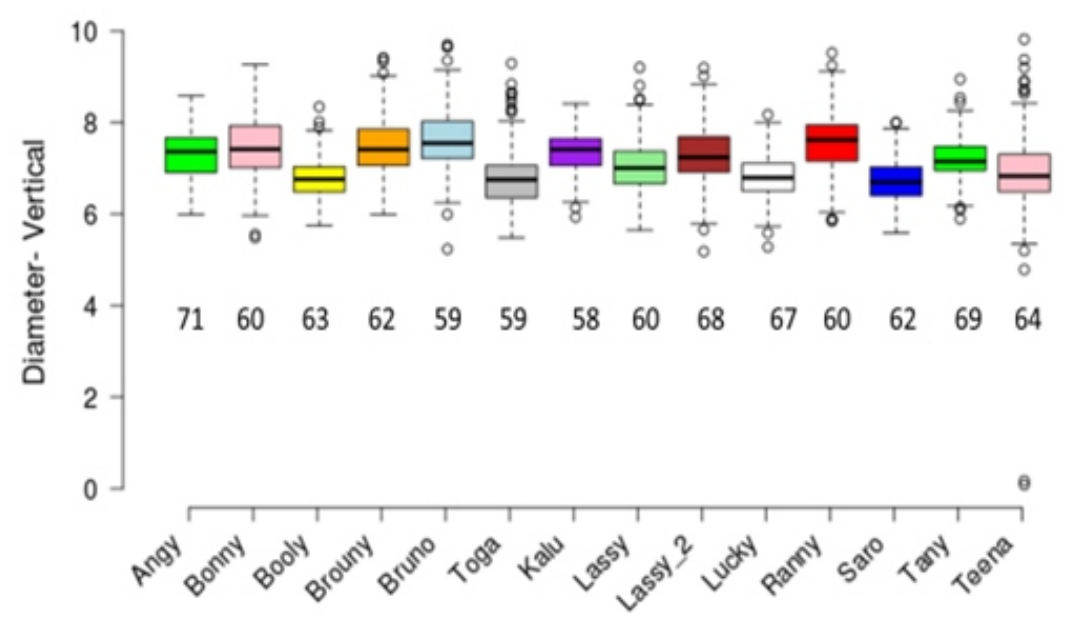

Figure 4. Variation of vertical diameter of red blood cells with MCV

stable and accurate, however, random errors can be caused by preanalytical and postanalytical problems, rather than by instrument or reagent problems, resulting in changes in one or more parameters tested which cannot be adequately explained by changes in the patient's clinical condition (Houwen, 1989). Preanalytical errors include specimen collection, improper specimen handling and labeling, or misidentification of the patient, while postanalytical errors include failure to correct test values for dilution, transcription errors, and misinterpretation of test results (Houwen, 1989). Transcription errors in automated hemoanalyzers can occur at a rate of approximately 1 per 500 samples or more, while the frequency of random errors in most laboratories exceeds 1 per 200 to 500 samples (Houwen, 1989). Therefore, a blood smear must be examined if and when an automated instrument produces highly improbable results which may be factitious (Bain, 2005). Future morphometric studies are warranted for further investigations on the relationship between these parameters.

\section{CONCLUSION}

Morphometry using a Laser scanning confocal microscope and ZEN lite (blue edition) software from Carl Zeiss showed that diameters and surface area of canine erythrocytes did not correspond with the MCV values calculated by the automated analyzer, indicating that the MCV value has limitations as an objective measurement of detecting anisocytosis.

\section{REFERENCES}

Adili, N., Melizi, M., Belabbas, H., Bala, A., Merad, S., Bouali, F.,Bennoune, O. (2017).Morphometric study of Red blood cells in Sloughi and German shepherd dog s.Bulgarian Journal of Veterinary Medicine, 20(2): 125-130.

Albertini, M.C., Teodori, L., Piatti, E., Piacentini, M.P., Accorsi, A.,Rocchi, M.B.(2003). Automated analysis of morphometric parameters for accurate definition of erythrocyte cell shape. Cytometry part A, 52(1): 12-18.

Bain, B.J. (2005). Diagnosis from the blood smear. New England Journal of Medicine, 353(5): 498-507.
Bernhard, G., Martin, G. (2012).Formation, Optical Image, and Electronic Signal Processing.Microscopy from Carl Zeiss / Sources.

Cortada (2013). Information about the software ZEN 2012 Black edition: 14.ISBN: 4101381005120

Ford, J. (2013). Red blood cell morphology, International Journal of Laboratory Hematology, 35, 351357.

Ghasemi, A.,Zahediasl, S. (2012). Normality tests for statistical analysis: a guide for non-statisticians. International Journal of Endocrinology and Metabolism, 10(2): 486.

Greer, J.P. (2009). Examination of the Blood and Bone marrow. In: Wintrobe's Clinical Hematology, Chapter 1.https://books.google.lk/books?isbn=0781765072.

Houwen, B. (1989). Delta checks for random error detection in hematology tests. Https://academic.oup.com/ labmed/article-pdf/20/6/410/8267391/labmed20-0410.pdf

Jain, N.C. (1993). Chapters 1 and 7: Essentials of Veterinary Hematology. Lea \&Feiger, Philadelphia.

Jay, A.W.L.,Rowlands,S. (1975). The stages of osmotic haemolysis. Journal of Physiology, 252, 817-832

Perry, D.J., Lowe, D.A.S., Wilson, C.I.D., Harris, R.1., Stevens, M.J. (1985). The red cell distribution width in macrocytosis. British Journal of Haematology, 61:559 (Abstract).

Poljičak-Milas, N., Kardum-Skelin, I., Vuđan, M., Silvija Marenjak, T., Ballarin-Perharić,A., Milas, Z., (2009).Blood cell count analyses and erythrocyte morphometry in New Zealand white rabbits. Veterinarskiarhiv, 79(6): 561-571.

Proctor, S.J., Cox, J.R., Sheridan, T.J. (1976). Anisocytosis and the C-1000 Channelyzer in miacrocyticanaemia. Journal of Clinical Pathology, 29: 719-723

Silva, I and Mallawa, M.R.C.K. (2010). A monograph on Clinical Hematology and Veterinary Diagnostic Techniques, Department of Veterinary Clinical Sciences, University of Peradeniya, Sri Lanka.

Thrall, M.A. (2006).Veterinary Hematology and Clinical Chemistry.Blackwell Publishing.

Quality control (qc) information and troubleshooting guide Hematology (2013). Https://www.beckmancoulter.com/ ucm/idc/groups/public/.../glb_bci_153627.pdf

Udroiu, I., (2014). Estimation of erythrocyte surface area in mammals, Department di Science, University Degili Studi Roma XIV: 1403.7660.

Weiss, D.J. and Wardrop, K.J. (2011). Eds., Schalm's veterinary hematology ( $6^{\text {th }}$ Edition $)$, John Wiley \& Sons. 